\title{
Museumsrezension
}

\section{Das Musée des Arts et Métiers in Paris}

\section{Eine dreidimensionale Enzyklopädie}

\author{
Von Gerhard Dohrn-Van Rossum
}

Spektakulär ist schon der Zugang zum neuen Musée des Arts et Métiers in Paris. Die gleichnamige Metro-Station präsentiert sich als eine riesige, aus Kupferplatten genietete Röhre mit messingnen Bullaugen. In den schmalen Schlitzen für das Tageslicht hängen Gestänge und Übersetzungen einer unsichtbaren Dampfmaschine. Ein ungewöhnlich eleganter Bahnhof im Look des UBoots Nautilus stimmt auf den Besuch des ältesten technischen Museums der Welt ein.

Seine Entstehung fällt in die Zeit des Terrors der Französischen Revolution. Am 31. August 1794 schlägt der Abbé Grégoire die Einrichtung eines „Conservatoire pour les arts et métiers, où se réuniront tous les outils et machines nouvellement inventés ou perfectionnés" vor. Vier Jahre später hatte er auch

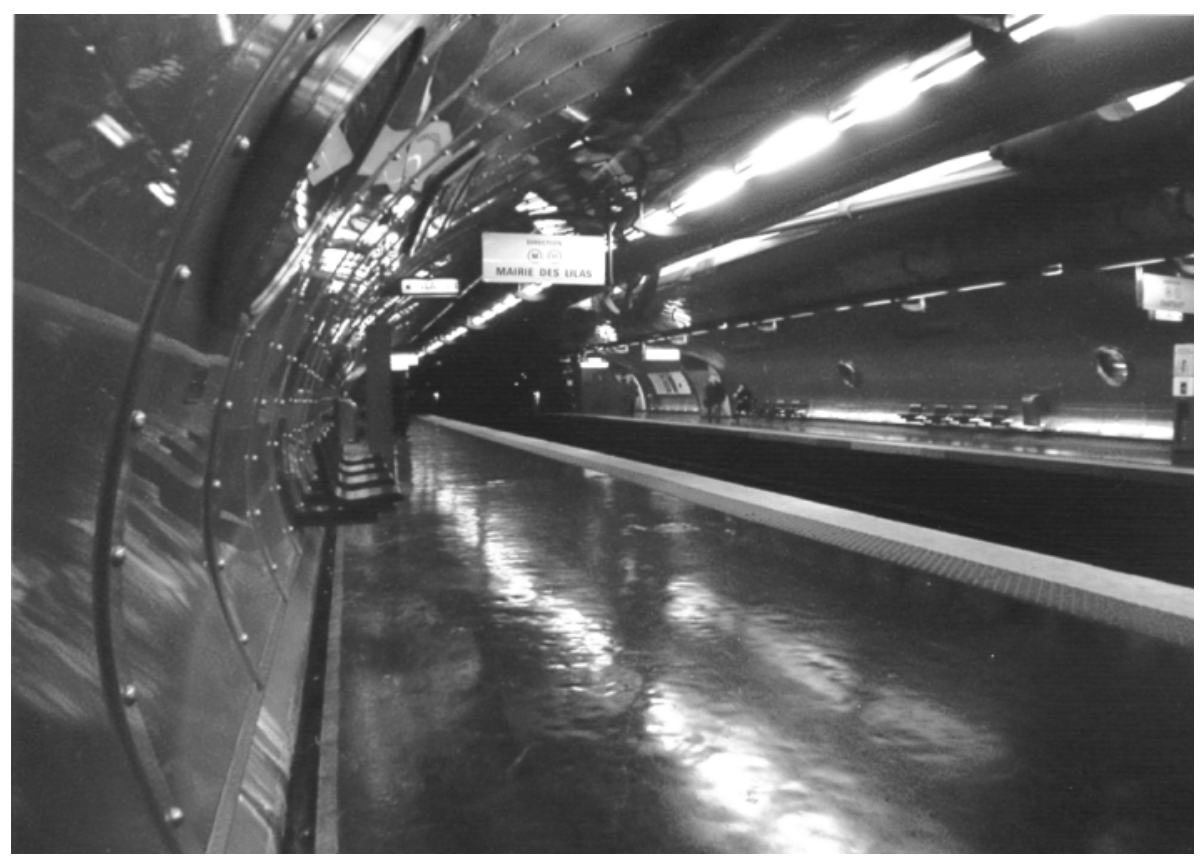

Abb. 1: Die Metro-Station „Ars et Métiers“ in Paris 
Baulichkeiten für das Vorhaben ausgemacht: das verlassene Kloster SaintMartin-des-Champs, dessen letzte Mönche der Guillotine zum Opfer gefallen waren. Das Kloster, am Standort einer frühmittelalterlichen Basilika, war nach der ersten Jahrtausendwende von den französischen Königen reich ausgestattet und in den folgenden Jahrhunderten vielfach ausgebaut worden. Hier sollten nun ältere technische Sammlungen verschiedenster Provenienz, darunter die Automaten des Jacques Vaucanson aus königlichem Besitz, aber auch von Aristokraten konfiszierte Objekte, zusammengeführt und mit zeitgenössischen Maschinen, Werkzeugen, Modellen, Zeichnungen und Büchern zur Förderung der nationalen Gewerbe und Industrien vereinigt werden. Der menschliche Fortschritt, als Perfektionierung und als Invention, sollte durch Bereitstellung enzyklopädischer und allgemein zugänglicher Information auch in Form von materiellen Objekten auf Dauer dargestellt werden. Gleichzeitig suchte man Kontakt zu den Ingenieuren und Konstrukteuren der allerneuesten Textilmaschinen, Raddampfer und Lokomobilen. Nicolas Cugnots gewaltiger Dampfwagen gehörte zu den ersten für das neue Domizil requirierten Objekten. Dem 1802 eröffneten Institut wurden eine Textilgewerbe- und eine Zeichenschule angegliedert, und bald wurden auch Lehrstühle eingerichtet. Nach amerikanischem Vorbild war in Frankreich der Erfinderschutz 1791 gesetzlich verankert worden, und bis zum Beginn des 20. Jahrhunderts prüfte das Institut als technisches Laboratorium auch die Patentgesuche (brevets d'invention) für Frankreich. Aus dem Benediktinerkloster war mehr als ein Museum, war ein nationaler Tempel der Technologie geworden.

Das Conservatoire national des arts et métiers (CNAM) ist heute eine Großorganisation zur technischen Aus- und Weiterbildung eines breiten Interessentenkreises und der Technologieförderung. Der Lehrstuhl für Technikgeschichte, von dem in den letzen Jahrzehnten wichtige Forschungsinitiativen ausgegangen sind, unterhält seit 1987 in Zusammenarbeit mit anderen Instituten ein Graduierungsprogramm mit einem Abschluss (DEA) für Geschichte der Technik. Ein aktuell interessantes Programm ist das Conservatoire numérique des Arts \& Métiers (Cnum), das eine digitalisierte Bibliothek zur Technik- und Wissenschaftsgeschichte einrichtet.

Schon in der Zwischenkriegszeit hatten die inzwischen riesigen Sammlungen eher den Charakter eines Depots angenommen, und nach dem Krieg war das Museum, auch wenn manche erfolgreiche Ausstellung veranstaltet worden ist, verwahrlost und die Exponate verstaubt. Das Museum geriet überdies in eine aussichtslose Konkurrenz zu modern konzipierten neuen Projekten, zunächst zum 1937 eingerichteten Palais de la Découverte und dann zu der auf dem gewaltigen aufgelassenen Schlachthofgelände im Park de La Villette 1986 eröffneten Cité de Sciences et de l'Industrie. Der Neukonzeption des Museums war damit der Weg gewiesen: respektvolle Rekonstruktion und Erneuerung eines alten Museums mit denkmalgeschützten Baulichkeiten. Der nach einem Jahrzehnt der Renovierung folgenden offiziellen Eröffnung im Jahr 2000 
war 1994 die international viel beachtete Neueröffnung der Metro-Station „Arts et Métiers" vorausgegangen, die architektonisch vom U-Boot Nautilus aus der Verfilmung des Romans 20.0000 Meilen unter dem Meer (1954) von Jules Verne inspiriert ist.

Der hier nur in Stichworten anzugebende Rundgang durch die Abteilungen beginnt in der Sammlung wissenschaftlicher Instrumente (Metrologie, Zeitmesstechnik, Pascalsche Rechenmaschine von 1642). Zentrum der Abteilung Materialien ist das Zyklotron des Collège de France aus dem Jahr 1937. Die Abteilung Bautechnik zeigt eine faszinierende Modellsammlung von Treppenkonstruktionen, Baumaschinen und Bauten bis in die Zeit des Stahlbaues. Einer Abteilung Kommunikation mit optischen Geräten, Kameras, Schreib- und Telegrafenmaschinen folgt eine Abteilung Energie mit Mühlen, Wasserhebewerken, Turbinen und Generatoren. Der Abteilung Mechanik mit ihren Maschinenmodellen ist das mehrmals täglich in Betrieb genommene Automatentheater angegliedert. Über dem Eingang zur räumlich größten Abteilung Transport hängt die vogelähnliche Flugmaschine von Clément Ader. Im hohen Gewölbe der alten Kirche hängt zentral das Foucaultsche Pendel zur Veranschaulichung der Erdrotation neben anderem Fluggerät. Im Kirchraum ist ein mehrstöckiges

Abb. 2: Flugmaschine von Clément Ader in der Abteilung Transport

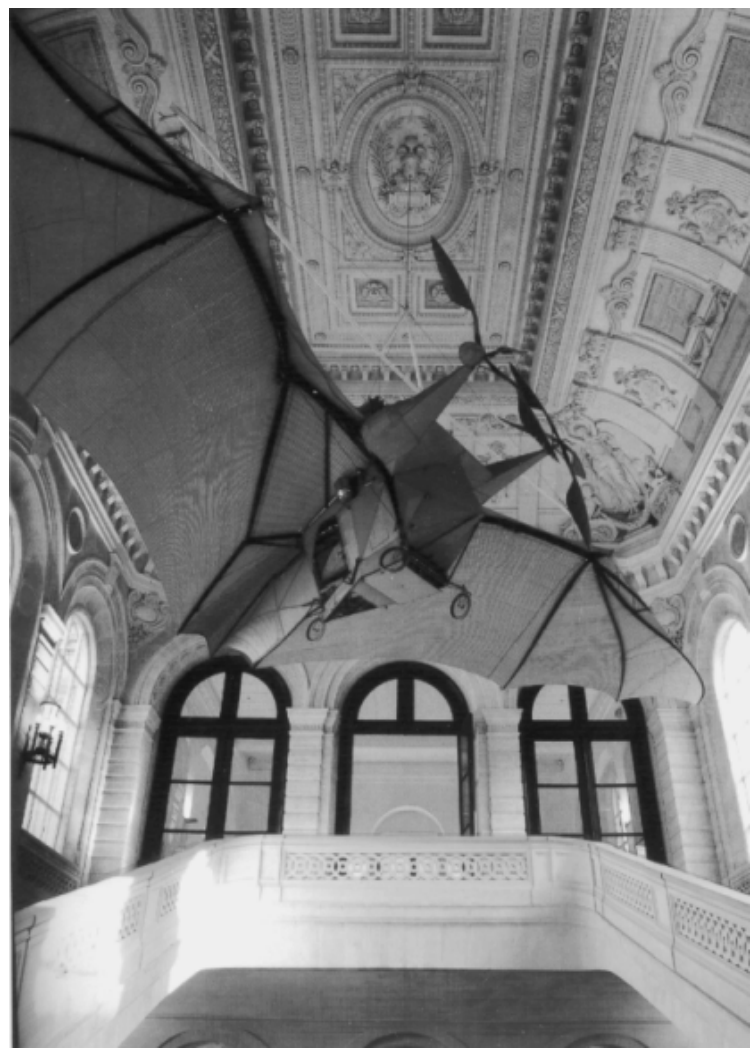




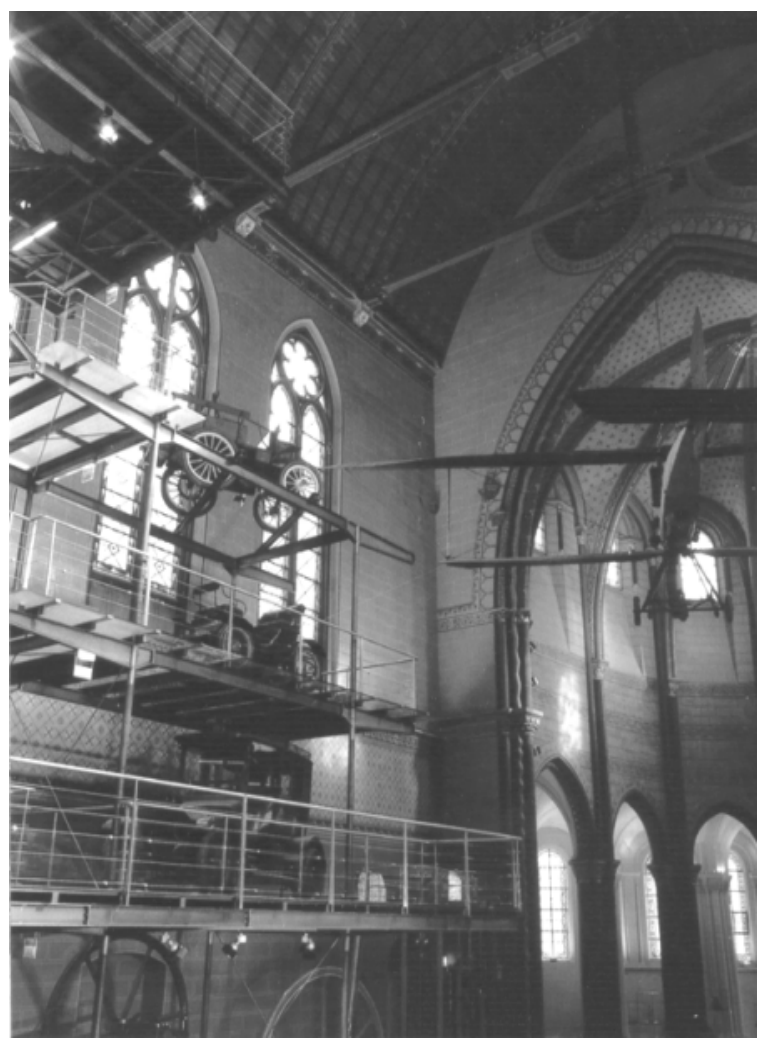

Abb. 3: Mehrstöckiges Metallgerüst mit Automobilen in der Abteilung Transport

Metallgerüst mit Lokomotiven und Automobilen bestückt. Auch wenn gelegentlich und sehr dezent audiovisuelle Hilfsmittel Funktionen und Abläufe erläutern, hat das neue Museum seinen historischen Charakter bewahrt. Artefakte und kunstvolle Modelle werden in hölzernen Vitrinen präsentiert. Es bewegt sich wenig, zu hören ist kaum etwas. Es ist kein Museum für Kinder, wohl aber für interessierte Jugendliche. Das ästhetische Vergnügen hat eine deutliche nostalgische Komponente. Die so genannte Wirklichkeit der industriellen Produktion ist hier nicht Thema, sondern ganz überwiegend die nachvollziehbare und anschauliche Rekonstruktion technischer Abläufe.

Gegen kritische Einwände, dass solche Museen Bewahranstalten technischer Relikte seien, dass sie den Wandel der Erwerbsarbeit, die soziale Frage, die Globalisierung und die Genderfragen außer acht ließen, sei hier nicht durch Hinweise auf einige - wenige - hoch moderne Exponate oder zeitgemäße Formen der Benutzerführung geantwortet. Solche Ansprüche werden gar nicht erhoben und wären in den historischen Baulichkeiten auch gar nicht zu realisieren. Der Vorteil der Metropole Paris besteht auch darin, dass andere Museen vom Typ der Cité des Sciences et de l'Industrie sich diesen Herausforderungen erfolgreich stellen. Dieses neue alte Museum macht dagegen absichtslos 
auch die Geschichte der Musealisierung von Technik in den letzten zwei Jahrhunderten zum Thema. Die früher hohe Bedeutung von ausgestellten Erzeugnissen, aber auch von Modellen für die Stimulierung von Innovation wie auch für ganz praktische Ausbildungszwecke wird unmittelbar anschaulich. Die Gebäude wie die Exponate verdeutlichen auch, dass der Fokus auf eine - soweit es das gibt - nationale Technikgeschichte in mancher Hinsicht auch sinnvoll sein kann. Man erfährt z. B. im Chor einer von der Revolution säkularisierten Kirche, dass nicht nur die Erklärung der Menschenrechte von Frankreich aus exportiert, sondern dass auch die Freiheitsstatue vor New York in Frankreich entworfen und vorproduziert worden ist. Das Musée des Arts et Métiers in Paris war in der Zeit der Industriellen Revolution ebenso wie Jules Vernes' 1870 erschienener Zukunftsroman fraglos Ausdruck eines noch ungebrochenen Vertrauens in den technischen Fortschritt. Ebenso wie die Nautilus als Symbol für dieses Vertrauen überdauert hat und unvermindert zu einer Beschäftigung mit der Geschichte der Technik einlädt, eignet sich dieses Museum als Einladung und Aufforderung.

Das Musée des Arts et Métiers im Internet: http://www.arts-et-metiers.net/ 
